\title{
Optimization under uncertainty: state-of-the-art and opportunities
}

\author{
Nikolaos V. Sahinidis* \\ Department of Chemical and Biomolecular Engineering, University of Illinois, 600 South Mathews Avenue, Urbana, IL 61801, USA
}

\begin{abstract}
A large number of problems in production planning and scheduling, location, transportation, finance, and engineering design require that decisions be made in the presence of uncertainty. Uncertainty, for instance, governs the prices of fuels, the availability of electricity, and the demand for chemicals. A key difficulty in optimization under uncertainty is in dealing with an uncertainty space that is huge and frequently leads to very large-scale optimization models. Decision-making under uncertainty is often further complicated by the presence of integer decision variables to model logical and other discrete decisions in a multi-period or multi-stage setting.

This paper reviews theory and methodology that have been developed to cope with the complexity of optimization problems under uncertainty. We discuss and contrast the classical recourse-based stochastic programming, robust stochastic programming, probabilistic (chance-constraint) programming, fuzzy programming, and stochastic dynamic programming. The advantages and shortcomings of these models are reviewed and illustrated through examples. Applications and the state-of-the-art in computations are also reviewed.

Finally, we discuss several main areas for future development in this field. These include development of polynomial-time approximation schemes for multi-stage stochastic programs and the application of global optimization algorithms to two-stage and chance-constraint formulations.
\end{abstract}

(c) 2003 Elsevier Ltd. All rights reserved.

Keywords: Stochastic programming; Fuzzy programming; Stochastic dynamic programming; Global optimization; Approximation algorithms

\section{Introduction}

Over the second half of the 20th century, optimization found widespread applications in the study of physical and chemical systems, production planning and scheduling systems, location and transportation problems, resource allocation in financial systems, and engineering design. From the very beginning of the application of optimization to these problems, it was recognized that analysts of natural and technological systems are almost always confronted with uncertainty.

This paper grew out of an earlier work (Sahinidis, 2003) with the main purpose to provide a short overview of optimization under uncertainty. It is beyond the scope of the paper to provide a detailed coverage of the field. Instead, we will give pointers to the literature that can be used as starting points for further study. For additional details and information, we refer the reader to the recent textbooks of Bertsekas and Tsitsiklis (1996), Birge and Louveaux (1997),

* Tel.: +1-217-244-1304; fax: +1-217-333-5052.

E-mail address: nikos@uiuc.edu (N.V. Sahinidis).
Kall and Wallace (1994), Prékopa (1995), and Zimmermann (1991), and the very informative Stochastic Programming Community Home Page (2003).

Beginning with the seminal works of Beale (1955), Bellman (1957), Bellman and Zadeh (1970), Charnes and Cooper (1959), Dantzig (1955), and Tintner (1955), optimization under uncertainty has experienced rapid development in both theory and algorithms. Today, Dantzig still considers planning under uncertainty as one of the most important open problems in optimization (Horner, 1999).

Approaches to optimization under uncertainty have followed a variety of modeling philosophies, including expectation minimization, minimization of deviations from goals, minimization of maximum costs, and optimization over soft constraints. The paper begins with an overview of the main approaches to optimization under uncertainty: stochastic programming (recourse models, robust stochastic programming, and probabilistic models), fuzzy programming (flexible and possibilistic programming), and stochastic dynamic programming. Then, we review applications and the state-of-the-art in computations, as well as important algorithmic developments by the process systems engineering community. Finally, we draw connections 
between models for optimization under uncertainty and global optimization. Throughout the presentation, we point out the fundamental differences of different modeling philosophies in optimization under uncertainty.

\section{Stochastic programming}

\subsection{Programming with recourse}

Under the standard two-stage stochastic programming paradigm, the decision variables of an optimization problem under uncertainty are partitioned into two sets. The firststage variables are those that have to be decided before the actual realization of the uncertain parameters. Subsequently, once the random events have presented themselves, further design or operational policy improvements can be made by selecting, at a certain cost, the values of the second-stage, or recourse, variables. Traditionally, the second-stage variables are interpreted as corrective measures or recourse against any infeasibilities arising due to a particular realization of uncertainty. However, the second-stage problem may also be an operational-level decision problem following a first-stage plan and the uncertainty realization. Due to uncertainty, the second-stage cost is a random variable. The objective is to choose the first-stage variables in a way that the sum of the first-stage costs and the expected value of the random second-stage costs is minimized. The concept of recourse has been applied to linear, integer, and non-linear programming.

\subsubsection{Stochastic linear programming}

A standard formulation of the two-stage stochastic linear program is (cf. Birge \& Louveaux, 1997; Kall \& Wallace, 1994):

$\min c^{t} x+E_{\omega \in \Omega}[Q(x, \omega)], \quad$ s.t. $\quad x \in X$,

with

$Q(x, \omega)=\min f(\omega)^{t} y$,

s.t. $\quad D(\omega) y \geq h(\omega)+T(\omega) x, \quad y \in Y$,

where $X \subseteq \mathfrak{R}^{n_{1}}$ and $Y \subseteq \mathfrak{R}^{n_{2}}$ are polyhedral sets. Here, $c \in \Re^{n_{1}}, \omega$ is a random variable from a probability space $(\Omega, \mathcal{F}, \mathcal{P})$ with $\Omega \subseteq \mathfrak{R}^{k}, f: \Omega \rightarrow \mathfrak{R}^{n_{2}}, h: \Omega \rightarrow \mathfrak{R}^{m_{2}}, D:$ $\Omega \rightarrow \Re^{m_{2} \times n_{2}}, T: \Omega \rightarrow \mathfrak{R}^{m_{2} \times n_{1}}$. Problem (1) with variables $x$ constitute the first stage which needs to be decided prior to the realization of the uncertain parameters $\omega \in \Omega$. Problem (2) with variables $y$ constitute the second stage.

Under the assumption of discrete distributions of the uncertain parameters, the problem can be equivalently formulated as a large-scale linear program which can be solved using standard linear programming technology. Convexity properties of the recourse function $Q(\cdot)$ (Wets, 1966, 1974) have been effectively used in decomposition-based solution strategies (Birge \& Louveaux, 1988; Van Slyke \& Wets,
1969). For continuous parameter distributions, these properties have been used to develop sampling-based decomposition and approximation schemes (Birge \& Louveaux, 1988; Higle \& Sen, 1991; Infanger, 1994; Ruszczyński, 1986; Shapiro \& Homem-de-Mello, 1998; Van Slyke \& Wets, 1969) as well as gradient-based algorithms (Ermoliev, 1983; Shapiro \& Wardi, 1996).

The two-stage formulation is readily extended to a multistage setting by modeling the uncertainty as a filtration process. Under discrete distributions, this reduces to a scenario tree of parameter realizations. Decomposition schemes that partition the time stage (Birge, 1985) as well as those that partition the scenario space (Rockafellar \& Wets, 1991) have been developed for multi-stage linear programs.

For an extensive discussion of stochastic linear programming, the reader is referred to standard textbooks on stochastic programming (Birge \& Louveaux, 1997; Infanger, 1994; Kall \& Wallace, 1994; Prékopa, 1995).

\subsubsection{Stochastic integer programming}

Stochastic integer programming addresses instances of (1) and (2), where the set $Y$ contains integer restrictions. Much of the early work in this area has been on the design and analysis of heuristics for two-stage stochastic integer programs (Dempster et al., 1981; Spaccamela, Rinnooy Kan, \& Stougie, 1984; Stougie, 1985). Exact algorithmic approaches are more recent and include extensions of the decomposition strategies for stochastic linear programs (Carøe \& Tind, 1998; Laporte \& Louveaux, 1993), Lagrangian relaxation schemes (Carøe \& Schultz, 1999; Takriti, Birge, \& Long, 1996), algebraic enumeration (Schultz, Stougie, \& van der Vlerk, 1998), convexification (Higle \& Sen, 2000; Sherali \& Fraticelli, 2002), and decomposition combined with branch-and-bound (Ahmed, Tawarmalani, \& Sahinidis, 2003).

For problems where the second-stage possesses a special structure known as simple recourse, Klein Haneveld, Stougie, and van der Vlerk $(1995,1996)$ proposed solution schemes based upon the construction of the convex hull of the second-stage value function. For more general recourse structures, Laporte and Louveaux (1993) proposed a decomposition-based approach for stochastic integer programs when the first-stage variables are pure binary. This restriction allows for the construction of optimality cuts that approximate the non-convex second-stage value function at only the binary first-stage solutions (but not necessarily at other points). The authors proposed a branch-and-bound algorithm to search the space of the first-stage variables for the globally optimal solution, while using the optimality cuts to approximate the second-stage value function. Finite termination of the algorithm is obvious since the number of first-stage solutions is finite. The method has been successfully used in solving two-stage stochastic location-routing problems (Laporte, Louveaux, \& Mercure, 1989, 1992, 1994; Laporte, Louveaux, \& van Hamme, 1994). Unfortunately, the algorithm is not applicable if any of the first-stage 
variables are continuous. Carøe and Tind (1998) generalized this algorithm to handle cases with mixed-integer firstand second-stage variables. The method requires the use of non-linear integer programming dual functions to approximate the second-stage value function in the space of the first-stage variables. The resulting master problem then consists of non-linear (possibly discontinuous) cuts and no practical method for its solution is currently known.

Carøe (1998), Carøe and Schultz (1999), and Carøe and Tind (1997) used the scenario decomposition approach of Rockafellar and Wets (1991) to develop a branch-and-bound algorithm for stochastic integer programs. This method solves the Lagrangian dual, obtained by dualizing the non-anticipativity constraints, as the lower bounding problem within a standard branch-and-bound framework. The subproblems of the Lagrangian dual correspond to the second-stage scenarios and are difficult to solve as they include integer constraints. Furthermore, although the Lagrangian dual provides very tight bounds, its solution requires the use of subgradient methods and is computationally expensive. A potential limitation of this approach is that finite termination is guaranteed only if the first-stage variables are purely discrete, or if an $\epsilon$-optimal termination criterion with $\epsilon>0$ is used.

Recently, Schultz et al. (1998) proposed a finite scheme for two-stage stochastic programs with discrete distributions and pure-integer second-stage variables. For this problem, Schultz et al. (1998) observe that only integer values of the right-hand side parameters of the second-stage problem are relevant. This fact is used to identify a finite set in the space of the first-stage variables containing the optimal solution. Schultz et al. (1998) propose complete enumeration of this set to search for the optimal solution. This set may be very large and evaluation of each of its elements requires the solution of second-stage integer subproblems. Thus, this approach is, in general, computationally prohibitive.

The above papers assume discrete probability distributions for the uncertain parameters. Except for simple cases that afford closed form solutions, sampling is required when dealing with continuous distributions of the problem parameters. Thus, convergence proofs for the resulting algorithms have to be probabilistic. For continuous distributions, Norkin, Ermoliev, and Ruszczyński (1998) developed a branch-and-bound algorithm that makes use of stochastic upper and lower bounds and proved almost sure convergence.

More recently, stochastic integer programming is receiving increased attention from the point of view of convexification. Higle and Sen (2000) and Sherali and Fraticelli (2002) have proposed algorithms that invoke ideas from lift-and-project (Balas, Ceria, \& Cornuèjols, 1993) and the reformulation-linearization technique (Adams \& Sherali, 1990) in the context of Benders-like decomposition approaches. These approaches are in their formative stages and no implementations have yet been reported. By exploiting some of the structural properties of stochastic integer programs, Ahmed et al. (2003) develop a finite branch-and-bound scheme for a class of stochastic integer programs and present some encouraging computational results on small problems.

\subsubsection{Stochastic non-linear programming}

Non-linear versions of the linear and integer programs considered in this paper have many applications, especially in engineering design, as well as planning and scheduling. For example, two-stage non-linear stochastic programming addresses the problem:

$\min f(x)+E_{\omega \in \Omega}[Q(x, \omega)], \quad$ s.t. $\quad g(x) \leq 0$,

with

$Q(x, \omega)=\min F(\omega, x, y), \quad$ s.t. $\quad G(\omega, x, y) \leq 0, \quad y \in Y$,

where $X \subseteq \mathfrak{R}^{n_{1}}, Y \subseteq \mathfrak{R}^{n_{2}}, \omega$ is a random variable from a probability space $(\Omega, \mathcal{F}, \mathcal{P})$ with $\Omega \subseteq \mathfrak{R}^{k}$, and the real functions $f, g, F$, and $G$ have conformable dimensions.

Most of the algorithms developed for stochastic linear programming carry over to the non-linear case. However, non-linearities may give rise to non-convexities and local optima. We refer the reader to the thesis of Bastin (2001) for a more detailed discussion of non-linear stochastic programming.

\subsubsection{Robust stochastic programming}

The recourse-based model (1) makes a decision based on present first-stage and expected second-stage costs, i.e., based on the assumption that the decision-maker is risk-neutral. To capture the notion of risk in stochastic programming, Mulvey, Vanderbei, and Zenios (1995) proposed the following modification of the objective function of (1):

$\min c^{t} x+E_{\omega \in \Omega}[Q(x, \omega)]+\lambda f(\omega, y)$

where $f$ is a variability measure, such as variance, of the second-stage costs and $\lambda$ is a non-negative scalar that represents the risk tolerance of the modeler. Large values of $\lambda$ result into solutions that reduce variance while small values of $\lambda$ reduce expected costs.

Applications of this, so-called robust stochastic programming, framework and its variants have been reported in power systems capacity expansion (Malcolm \& Zenios, 1994), power dispatch (Beraldi, Musmanno, \& Triki, 1998), chemical process planning (Ahmed \& Sahinidis, 1998), telecommunications network design (Bai, Carpenter, \& Mulvey, 1997; Laguna, 1998), and financial planning (Bai et al., 1997; Kouwenberg \& Zenios, 2001; Mulvey et al., 1995).

Various examples demonstrate that a straight forward deterministic reformulation of robust models may result in second-stage solutions that are suboptimal for the recourse problem (King, Takriti, \& Ahmed, 1997; Sen \& Higle, 1999). This is a highly undesirable property as it may lead to an underestimation of the recourse costs. Takriti 
and Ahmed (2003) proposed sufficient conditions on the variability measure to remedy this problem.

\subsection{Probabilistic programming}

The recourse-based approach to stochastic programming requires the decision-maker to assign a cost to recourse activities that are taken to ensure feasibility of the second-stage problem. In essence, the philosophy of this approach is that infeasibilities in the second stage are allowed at a certain penalty. The approach thus focuses on the minimization of expected recourse costs. In the probabilistic or chance-constraint approach, the focus is on the reliability of the system, i.e., the system's ability to meet feasibility in an uncertain environment. This reliability is expressed as a minimum requirement on the probability of satisfying constraints.

Consider the classical linear programming model:

$\max c^{t} x, \quad$ s.t. $\quad A x \geq b, \quad x \geq 0$,

where $c$ and $x$ are $n$-vectors, $b$ is an $m$-vector, and $A$ is an $m \times n$ matrix. Assume that there is uncertainty regarding the constraint matrix $A$ and the right-hand side vector $b$, and that the system is required to satisfy the corresponding constraint with a probability $p \in(0,1)$. Then, the probabilistic linear program corresponding to the classical (deterministic) linear program can be stated as follows:

$\max \quad c^{t} x, \quad$ s.t. $\quad P(A x \geq b) \geq p, \quad x \geq 0$.

Consider the case when $m=1$, i.e., the case of a single constraint $P\left(a^{t} x \geq b\right) \geq p$. Further, assume that the vector $a$ is deterministic while the right-hand side $b$ is a random variable with cumulative distribution $F$. Let $\beta$ be such that $F(\beta)=p$. Then, the constraint $P\left(a^{t} x \geq b\right) \geq p$ can be written as $F\left(a^{t} x\right) \geq p$ or $a^{t} x \geq \beta$. In this simple case, the probabilistic program is equivalent to a standard linear program.

For the case when the matrix $A$ is deterministic and the vector $b$ has a log-concave multivariate probability density function, Prékopa (1971) has shown that the feasible set of (3) is convex. Other standard cases in which probabilistic constraints can be converted to standard constraints are summarized in Prékopa (1995). However, in general, the feasible set of (3) may be non-convex. This issue is discussed later in this paper.

\section{Fuzzy mathematical programming}

Like stochastic programming, fuzzy programming also addresses optimization problems under uncertainty. A principal difference between the stochastic and fuzzy optimization approaches is in the way uncertainty is modeled. In the stochastic programming case, uncertainty is modeled through discrete or continuous probability functions. On the other hand, fuzzy programming considers random parameters as fuzzy numbers and constraints are treated as fuzzy sets. Some constraint violation is allowed and the degree of satisfaction of a constraint is defined as the membership function of the constraint. For example, consider a linear constraint $a^{t} x \leq \beta$ in terms of the decision vector $x$ and assume that the random right-hand side $\beta$ can take values in the range from $b$ to $b+\Delta b$, with $\Delta b \geq 0$. Then, the linear membership function, $u(x)$, of this constraint is defined as:

$u(x)= \begin{cases}1, & \text { if } a^{t} x \leq b, \\ 1-\frac{a^{t} x-b}{\Delta b}, & \text { if } b<a^{t} x \leq b+\Delta b, \\ 0, & \text { if } b+\Delta b<a^{t} x .\end{cases}$

Although other types of membership functions are also possible, the above linear membership function is typically used. Objective functions in fuzzy mathematical programming are treated as constraints with the lower and upper bounds of these constraints defining the decision maker's expectations.

Many of the developments in the area of fuzzy mathematical programming are based on the seminal paper by Bellman and Zadeh (1970). The field has been recently popularized by the work of Zimmermann (1991). Two types of fuzzy programming will be considered here: flexible programming and possibilistic programming. Flexible programming deals with right-hand side uncertainties while possibilistic programming recognizes uncertainties in the objective function coefficients as well as in constraint coefficients. In both types of fuzzy programming, the membership function is used to represent the degree of satisfaction of constraints, the decision-maker's expectations about the objective function level, and the range of uncertainty of coefficients.

\subsection{Flexible programming}

Consider the classical linear programming model:

$\max c^{t} x, \quad$ s.t. $\quad A x \leq b, \quad x \geq 0$,

where $c$ and $x$ are $n$-vectors, $b$ is an $m$-vector, and $A$ is an $m \times$ $n$ matrix. Let us suppose that there is uncertainty regarding the exact values of the coefficients and some violation of the constraints is acceptable within a certain range. This means that some parts of (4) can be fuzzy. When the elements of $A, b$, or $c$ are treated as fuzzy numbers rather than crisp numbers, constraints can be represented by fuzzy sets rather than by crisp inequalities, and objective functions can be represented by a fuzzy goal rather than a crisp objective function. We use $\tilde{\alpha}$ to indicate that the parameter $\alpha$ is fuzzy. Similarly, $a^{t} x \tilde{\leq} b$ means that $a^{t}$ should be essentially smaller than or equal to $b$, i.e., that this constraint is a soft constraint for which some violation is allowed. The tolerance or spread of the fuzzy parameter $\alpha$ will be denoted by $\Delta \alpha$.

A flexible programming problem can then be written as (Tanaka, Okuda, \& Asai, 1974; Zimmermann, 1991):

$\widetilde{\max } c^{t} x, \quad$ s.t. $A x \tilde{\leq} b, \quad x \geq 0$. 
Let us denote $\left(c_{j}, a_{1 j}, \ldots, a_{m j}\right)^{t}$ and $\left(v, b_{1}, \ldots, b_{m}\right)^{t}$ by $\left(\hat{a}_{0 j}, \hat{a}_{1 j}, \ldots, \hat{a}_{m j}\right)^{t}$ and $\left(\hat{b}_{0}, \hat{b}_{1}, \ldots, \hat{b}_{m}\right)^{t}$, respectively. Then, problem (5) can be rewritten as follows:

Find $x$, s.t. $\hat{A} x \tilde{\leq} \hat{b}$,

where $\hat{A}$ is an $(m+1) \times n$ matrix and $\hat{b}$ is an $(m+1)$ vector. It is assumed that the fuzzy constraints and fuzzy goal are subjectively defined by the decision-maker. Let $u_{i}(x)$ denote the membership for the $i$ th constraint of (5), $i=$ $1, \ldots, n$. Also, let $u_{0}(x)$ denote the membership function of the objective of (5). In addressing problem (6), Bellman and Zadeh (1970) define an optimal fuzzy decision to be:

$x^{*}=\arg \max _{x \geq 0} \min _{i=0, \ldots, n} u_{i}(x)$.

According to this definition, the optimal solution of problem (6) can be obtained by solving the non-linear programming problem:

$\max _{x \geq 0} \min _{i=0, \ldots, n} 1-\frac{\hat{A}_{i} x-b_{i}}{\Delta b_{i}}$.

By introducing one new variable $\lambda$, Zimmermann (1978) showed that, if all membership functions are linear, then (5) can be reduced to a classical linear program:

$\max \lambda, \quad$ s.t. $\quad \bar{A} x+\lambda \leq \bar{b}, \quad x \geq 0, \quad 0 \leq \lambda \leq 1$,

where the elements of $\bar{A}$ and $\bar{b}$ are $\bar{a}_{i j}=\hat{a}_{i j} / \Delta b_{i}$ and $\bar{b}_{i}=$ $1+\left(\hat{b}_{i} / \Delta b_{i}\right)$, respectively.

Problem (7) includes one more variable and one more constraint than the original problem (5). Although a linear membership function is only a very rough approximation of the knowledge of the decision-maker about the membership function, Delgado, Herrera, Verdegay, and Vila (1993) showed that the optimal solution obtained by using a linear membership function is often of the same quality as the solution obtained using a complicated non-linear membership function. Therefore, the use of linear membership provides an efficient way to solve fuzzy programs and obtain solutions of good quality.

Note that the spread of the objective function, $\Delta b_{0}$, must be provided by the decision-maker. It expresses the decision-maker's aspiration in regard to the objective function value. $\Delta b_{0}$ can be estimated as the difference of the potential upper and lower bounds for the objective function (Zimmermann, 1991).

\subsection{Possibilistic programming}

When (4) involves uncertainty in constraint coefficients, the fuzzy program is called possibilistic (Tanaka \& Asai, 1984). A possibilistic linear programming problem can be written as follows:

$\widetilde{\max } \tilde{c}^{t} x, \quad$ s.t. $\quad \tilde{A} x \leq \tilde{b}, \quad x \geq 0$.

Let $a_{i j}$ and $\Delta a_{i j}$, respectively, represent the center and spread of the fuzzy number $\tilde{a}_{i j}$. Similarly, let $c_{j}$ and $\Delta c_{j}$ denote the center and spread of the fuzzy number $\tilde{c}_{j}$. Now, consider the following membership functions:

$u_{i}(x)= \begin{cases}1, & \text { if } A_{i} x \leq b_{i}, \\ 1-\frac{A_{i} x-b_{i}}{\Delta A_{i} x+\Delta b_{i}}, & \text { if } b_{i}<A_{i} x<b_{i}+\Delta A_{i} x+\Delta b_{i}, \\ 0, & \text { otherwise, }\end{cases}$

and

$u_{0}(x)= \begin{cases}1, & \text { if } b_{0} \leq c^{t} x, \\ 1-\frac{b_{0}-c^{t} x}{\Delta b_{0}+\Delta c^{t} x}, & \text { if } b_{0}-\Delta b_{0}-\Delta c^{t} x<c x<b_{0}, \\ 0, & \text { otherwise, }\end{cases}$

where $\left[b_{0}-\Delta b_{0}, b_{0}\right]$ denotes the aspiration range for the objective. Then, the Bellman-Zadeh decision-making criterion leads to the following equivalent of the possibilistic program after the introduction of a new variable $\lambda$ :

$\max \quad \lambda, \quad$ s.t. $\quad c^{t} x+\Delta c^{t} x(1-\lambda) \geq b_{0}-\Delta b_{0}(1-\lambda)$,

$A x-\Delta A x(1-\lambda) \leq b+\Delta b(1-\lambda), \quad x \geq 0, \quad 0 \leq \lambda \leq 1$.

The possibilistic programming problem (8) has been reduced into the non-linear programming problem (9). Here, $b_{0}$ and $\Delta b_{0}$ can be calculated by interval linear programming (Tong, 1994). In general, (9) has a non-convex feasible space.

\section{Stochastic dynamic programming}

Dynamic programming is the termed coined by Bellman to describe his mathematical theory of dealing with multi-stage decision processes (Bellman, 1957). From the very beginning, uncertainty was recognized as an integral part of the dynamic environment. Consider a discrete-time system that evolves over $N$ time periods. At time instant $k$, we use $x_{k}$, $u_{k}$, and $w_{k}$, to denote, respectively, the state of the system, a control action, and a random parameter such as disturbance or noise. It is assumed that, in period $k$, the present state of the system is fully determined by its recent history:

$x_{k}=f_{k-1}\left(x_{k-1}, u_{k-1}, w_{k-1}\right), \quad k=1, \ldots, N$,

while $u_{k}$ is selected with knowledge of the present state from a set of allowable control actions:

$u_{k} \in U_{k}\left(x_{k}\right)$,

and the uncertainty $w_{k}$ follows some distribution that depends only on the current state and control action:

$P_{w_{k}}\left(x_{k}, u_{k}\right)$.

The decision-maker wishes to minimize an additive cost function over the entire time horizon:

$\min _{u_{k} \in U_{k}\left(x_{k}\right), k=0, \ldots, N-1} E_{w}\left\{g_{N}\left(x_{N}\right)+\sum_{k=0}^{N-1} g\left(x_{k}, u_{k}, w_{k}\right)\right\}$. 
Consider now the tail subproblem of minimizing the cost-to-go from time $i$ to time $N$ :

$\min _{u_{k} \in U_{k}\left(x_{k}\right), k=i, \ldots, N-1} E_{w}\left\{g_{N}\left(x_{N}\right)+\sum_{k=i}^{N-1} g\left(x_{k}, u_{k}, w_{k}\right)\right\}$.

Bellman's principle of optimality states that, no matter how we arrived at state $i$, the remaining decisions must be optimal for the tail subproblem. Dynamic programming first solves all tail subproblems of the final stage. Then, at a generic step $k=N-2, \ldots, 0$, it solves all tail subproblems of length $N-k$ using the solution of all the tail subproblems of shorter time length. Obviously, the original problem is solved at the last step of this process by utilizing the solutions of all tail subproblems.

Suitable algorithms must be employed to solve the tail problems. For example, non-linear or other stochastic programming algorithms may be invoked. In addition, as all tail subproblems must be solved by the algorithm, the procedure is very intensive computationally and suffers from the curse of dimensionality as the computational time and storage requirements grow exponentially in the number of state and control variables. These difficulties have led to the development of several approximation techniques, including the approximation of the optimal cost-to-go function by that of a related simpler problem, through simulation, or through a heuristic learning scheme such as neuro-dynamic programming. The class of neuro-dynamic programming algorithms, in particular, combines techniques from reinforcement learning to systematically approximate the cost-to-go functions in order to obtain suboptimal policies for dynamic programming problems (Bertsekas \& Tsitsiklis, 1996).

\section{Applications and computations}

\subsection{Applications of stochastic programming}

The original applications of stochastic programming included agricultural economics in Iowa under land and labor constraints (Tintner, 1955), the allocation of aircraft to routes with penalties for lost passengers (Ferguson \& Dantzig, 1956), and the production of heating oil with constraints on meeting sales and not exceeding capacity (Charnes, Cooper, \& Symonds, 1958). More recent applications have included:

- production planning (Bitran, Haas, \& Matsuo, 1986; Dempster et al., 1981; Escudero, Kamesam, King, \& Wets, 1993; Lenstra, Rinnooy Kan, \& Stougie, 1983);

- scheduling (Birge \& Dempster, 1996; Dempster, 1982; Dempster et al., 1983; Tayur, Thomas, \& Natraj, 1995);

- routing (Laporte et al., 1989, 1992; Spaccamela et al., 1984);

- location (Laporte et al., 1994);
- capacity expansion (Ahmed, King, \& Parija, 2003; Berman, Ganz, \& Wagner, 1994; Bienstock \& Shapiro, 1988; Davis, Dempster, Sethi, \& Vermes, 1987; Eppen, Martin, \& Schrage, 1989; Malcolm \& Zenios, 1994; Sherali, Soyster, Murphy, \& Sen, 1984);

- energy investment and electricity production (Carøe, Ruszczyński, \& Schultz, 1997; Louveaux, 1980; Morton, 1996; Pereira \& Pinto, 1991; Takriti et al., 1996);

- environmental management and control (Birge \& Rosa, 1996; King, Rockafellar, Somlyody, \& Wets, 1988; Norkin et al., 1998; Pinter, 1991; Somlyody \& Wets, 1988; Wagner, Shamir, \& Marks, 1994; Watanabe \& Ellis, 1993);

- water management (Dupačová, Gaivoronski, Kos, \& Szantai, 1991);

- agriculture (Helgason \& Wallace, 1991; Shukla \& Gupta, 1989);

- telecommunications (Laguna, 1998; Tomasgard et al., 1998);

- design and optimization of chemical processing systems (Acevedo \& Pistikopoulos, 1998; Clay \& Grossmann, 1997; Gupta \& Maranas, 2000; Liu \& Sahinidis, 1996);

- finance (Carino \& Ziemba, 1998; Dert, 1995; Kallberg, White, \& Ziemba, 1982; Kouwenberg \& Zenios, 2001; Mulvey \& Vladimirou, 1992).

\subsection{Applications of fuzzy programming}

Applications of fuzzy programming have spanned an equally impressive number of diverse fields, and have included:

- production planning (Inuiguchi, Sakawa, \& Kum, 1994; Miller, Leung, Azhar, \& Sargent, 1997);

- transportation problems (Bit, Bisswal, \& Alam, 1993a, 1993b; Chalam, 1994; Chanas, Kolodziejczyk, \& Machaj, 1984; Chanas \& Kuchta, 1998; Li \& Lai, 2000; Sakawa, Nishizaki, \& Uemura, 2002);

- water supply planning and resource management (Nayak \& Panda, 2001; Slowinski, 1986; Wu, Huang, \& Guo, 1997);

- forest management (Pickenss \& Hof, 1991);

- capacity expansion (Liu \& Sahinidis, 1996, 1997b);

- bank management (Lai \& Hwang, 1993a, 1993b);

- portfolio selection (Parra, Terol, \& Uria, 2001);

- pattern classification (Nakayama \& Kagaku, 1998);

- environmental management (Chang, Chen, Shaw, \& Yang, 1997; Huang, Baetz, Huang, \& Liu, 2002).

\subsection{Applications of stochastic dynamic programming}

Applications of stochastic dynamic programming can be found essentially in all areas of multi-stage decision-making. Some recent applications have included:

- economics and finance (Tsitsiklis \& Van Roy, 1999; Van Roy \& Tsitsiklis, 2001; Zimmerman \& Carter, 2003); 
- design of experiments (Ben-Gal \& Caramanis, 2002);

- biological and agricultural sciences (Kirby, Fiksen, \& Hart, 2000; Richter et al., 2003; Sernland, Olsson, \& Holmgren, 2003; Yalcin \& Stott, 2000);

- production planning (Cheng, Subrahmanian, \& Westerberg, 2003), scheduling (Bertsekas \& Castañon, 1999; Mo, Gjelsvik, \& Grundt, 2001; Shen \& Leachman, 2003), and supply chain management (Bitran, Caldentey, \& Mondschein, 1998);

- airline management (Chen, Gunther, \& Johnson, 2003);

- environmental management (Danielsson, 2002);

- workforce management (Anderson, 2001).

\subsection{State-of-the-art in computations}

As of the time of this writing, there do not exist any widely-available general-purpose software implementations of fuzzy mathematical programming or stochastic dynamic programming. In certain instances, these methodologies lead to formulations that can be solved with traditional mathematical programming software. In other cases, their implementation requires problem-specific algebraic manipulations that make difficult the development of general-purpose software. On the other hand, commercial software for stochastic programming have recently emerged (IBM Stochastic Solutions, 2002; Infanger, 2003).

The purpose of this subsection is to discuss what might be within the capabilities of stochastic programming algorithms and software. Stochastic programs are much more difficult than their deterministic counterparts. Yet, significant progress has been made towards their exact and approximate solution.

Exact solution of deterministic equivalents of stochastic linear programs relies on decomposition. In a recent review paper, Birge (1997) reports the exact solution, on parallel computers, of stochastic linear programs with up to one million variables in their deterministic equivalents. Much larger problems are typically solvable by sampling-based rather than decomposition methods. Impressive computational results on a computational grid are reported by Linderoth, Shapiro, and Wright (2002) on stochastic linear programs with up to $10^{81}$ scenarios. These problems were solved using sample-average approximations.

Much smaller stochastic programs have been reported solved for the integer case. Exact solutions have been recently obtained for relatively small problems as reported by Ahmed et al. (2003). We refer the reader to Verweij, Ahmed, Kleywegt, Nemhauser, and Shapiro (2002) for a recent application of sampling-based methods to a stochastic routing problem with $2^{1694}$ scenarios within an estimated $1 \%$ of optimality.

Finally, we refer the reader to the Stochastic Programming Community Home Page (2003) for links to software as well as test problem collections for stochastic programming.

\section{Developments by the process systems engineering community}

The process systems engineering community has long been involved in the development of tools for the solution of design and operational problems under uncertainty. These efforts have been motivated by applications and, in many cases, yielded general-purpose algorithms. In this section, we review some of these developments.

\subsection{Flexibility analysis and optimization}

Considerable effort has been devoted to design and operational problems under uncertainty where the objective is to identify or maximize the flexibility, which is defined as the range of uncertain parameters that can be dealt with by a specific design or operational plan. Systematic optimization tools have been developed for measuring flexibility and analyzing the trade-offs between cost and flexibility (cf. Bansal, Perkins, \& Pistikopoulos, 1998; Floudas, Gümüç, \& Ierapetritou, 2001; Pistikopoulos \& Grossmann, 1989a, 1989b; Pistikopoulos and Mazzuchi, 1990; Straub \& Grossmann, 1993; Swaney \& Grossmann, 1985).

\subsection{Aggregation-disaggregation algorithm for two-stage stochastic linear programming}

Clay and Grossmann (1997) address the two-stage stochastic linear programming program with discrete probability distributions for the uncertain parameters. In recognition of the fact that the complexity of the problem stems from the large number of scenarios of uncertainty, these authors propose an aggregation of the probability space followed by successive disaggregation. Lower and upper bounds on the optimal objective function of the original problem were then developed over partition elements of the probability space. A sensitivity analysis was also developed for guiding the disaggregation process. The algorithm was applied to stochastic planing models from the process industries and was demonstrated to require only few partitions for the bounds to converge. Problems with millions of rows and columns in the deterministic equivalent were successfully solved with this approach.

\subsection{Multiparametric programming approach for mixed-integer quadratic programming}

When the number of uncertain variables is relatively small, it is possible to obtain closed-form solutions of optimization problems in terms of the values of the uncertain parameters. Dua, Bozinis, and Pistikopoulos (2002) obtain such solutions for mixed-integer quadratic programs with a few uncertain parameters. The basic idea is to utilize parametric non-linear programming tools to systematically characterize the space of parameters by a set of regions of optimality. The algorithm developed by these 
authors was applied to model predictive and hybrid control problems.

In general, parametric programming algorithms can be used to obtain, in closed-form, the solution of an optimization problem as a function of a few uncertain parameters. Once uncertainty presents itself, the analytical solutions can be looked up to identify and implement the optimal strategy without a need to solve an optimization problem. This can lead to efficient on-line implementations.

\subsection{Exact branch-and-bound algorithm for two-stage stochastic integer programming}

Ahmed et al. (2003) address a general class of two-stage stochastic integer programs with integer recourse and discrete distributions. By restating the problem in terms of the so-called tender variables, the discontinuities associated with the value function of the second-stage integer problem become orthogonal to the variable axes. The authors then develop a branch-and-bound algorithm to solve this problem. This scheme departs from previous literature in that it avoids explicit enumeration of the search space while guaranteeing finiteness.

\subsection{An approximation scheme for multi-stage stochastic integer programs for capacity expansion}

In a recent line of research, Ahmed and Sahinidis (2000, 2003), Furman and Sahinidis (2003), and Liu and Sahinidis (1997a) proposed linear programming based heuristics for operational and design problems in process systems engineering. The distinguishing feature of this solution paradigm is the analytical derivation of bounds for the quality of the solutions obtained by heuristics that run in polynomial time. In particular, Ahmed and Sahinidis (2003) address the approximate solution of large-scale multi-stage stochastic integer programs arising from capacity expansion in the process industries. The presence of integer variables in every stage makes this problem very challenging. Through a suitable rounding of the linear programming relaxation and bundling of the capacity expansion decisions, these authors obtain a feasible integer solution to this problem. Through a probabilistic analysis, the authors prove that the optimality gap of the solution thus obtained almost surely vanishes asymptotically as the number of stages increases. Computational experience demonstrates that the proposed approach yields near-optimal solutions even for small problem sizes.

\section{Connections between global optimization and optimization under uncertainty}

The purpose of this section is to demonstrate that many optimization programs under uncertainty are very difficult to solve as they correspond to multi-extremal non-linear optimization problems even when this is not directly appar- ent, as is the case in seemingly linear formulations. For this purpose, we present two examples, the first from stochastic integer programming and the second from probabilistic programming. Other classes of stochastic programs that give rise to multiextremal global optimization problems are possibilistic programs (Liu \& Sahinidis, 1997b), stochastic programs with decision-dependent uncertainties (Ahmed, 2002), and robust stochastic programs (King et al., 1997). Recent developments in the area of global optimization (Tawarmalani \& Sahinidis, 2002) are thus very likely to play a major role towards the solution of optimization problems under uncertainty.

\subsection{Two-stage stochastic integer programming}

Consider the following four-scenario version of a two-stage stochastic integer program that has been considered by Schultz et al. (1998):

EX1: $\quad \min -1.5 x_{1}-4 x_{2}+\sum_{s=1}^{4} p^{s} Q^{s}\left(x_{1}, x_{2}\right)$,

s.t. $0 \leq x_{1} \leq 5, \quad 0 \leq x_{2} \leq 5$,

where $Q^{s}\left(x_{1}, x_{2}\right)$ is defined as the optimal objective function value of:

$$
\begin{aligned}
& \min \quad-16 y_{1}-19 y_{2}-23 y_{3}-28 y_{4}, \\
& \text { s.t. } \quad 2 y_{1}+3 y_{2}+4 y_{3}+5 y_{4} \leq \omega_{1}^{s}-\frac{1}{3} x_{1}-\frac{2}{3} x_{2} \quad 6 y_{1} \\
& +y_{2}+3 y_{3}+2 y_{4} \leq \omega_{2}^{s}-\frac{2}{3} x_{1}-\frac{1}{3} x_{2}, \quad y_{i} \in\{0,1\}, \\
& \\
& i=1, \ldots, 4,
\end{aligned}
$$

and $\left(\omega_{1}, \omega_{2}\right) \in\{5,15\} \times\{5,15\}$ with uniform probability (i.e., $p^{s}=1 / 4$ for $s=1, \ldots, 4$ ).

There are only two degrees of freedom in this example: the first-stage variables $x_{1}$ and $x_{2}$. Once their values are specified, the $y$-variables are determined by solving the second-stage integer optimization problem. This allows us to plot the objective function value of EX1 in the space of $x_{1}-x_{2}$ (Fig. 1 from Ahmed, 2002). The objective is piecewise polyhedral with several local minima. The unique global minimum is at $(0,3)$.

\subsection{Probabilistic programming}

Consider the following probabilistic program in terms of two variables and two probabilistic constraints:

EX2: $\quad \max \quad c^{t} x, \quad$ s.t. $\quad P\left(\begin{array}{c}x_{1}+x_{2} \geq b_{1} \\ x_{1}+3 x_{2} \geq b_{2}\end{array}\right) \geq 0.5$,

$x_{1} \geq 0 \quad x_{2} \geq 0$,

where $b_{1}$ and $b_{2}$ are dependent random variables with $P\left(b_{1}=2, b_{2}=4\right)=0.5$ and $P\left(b_{1}=3, b_{2}=0\right)=0.5$. Clearly, any $\left(x_{1}, x_{2}\right)$ satisfying $x_{1}+x_{2} \geq 2$ and $x_{1}+3 x_{2} \geq 4$ is feasible to EX2. Let this polyhedral set be denoted by 


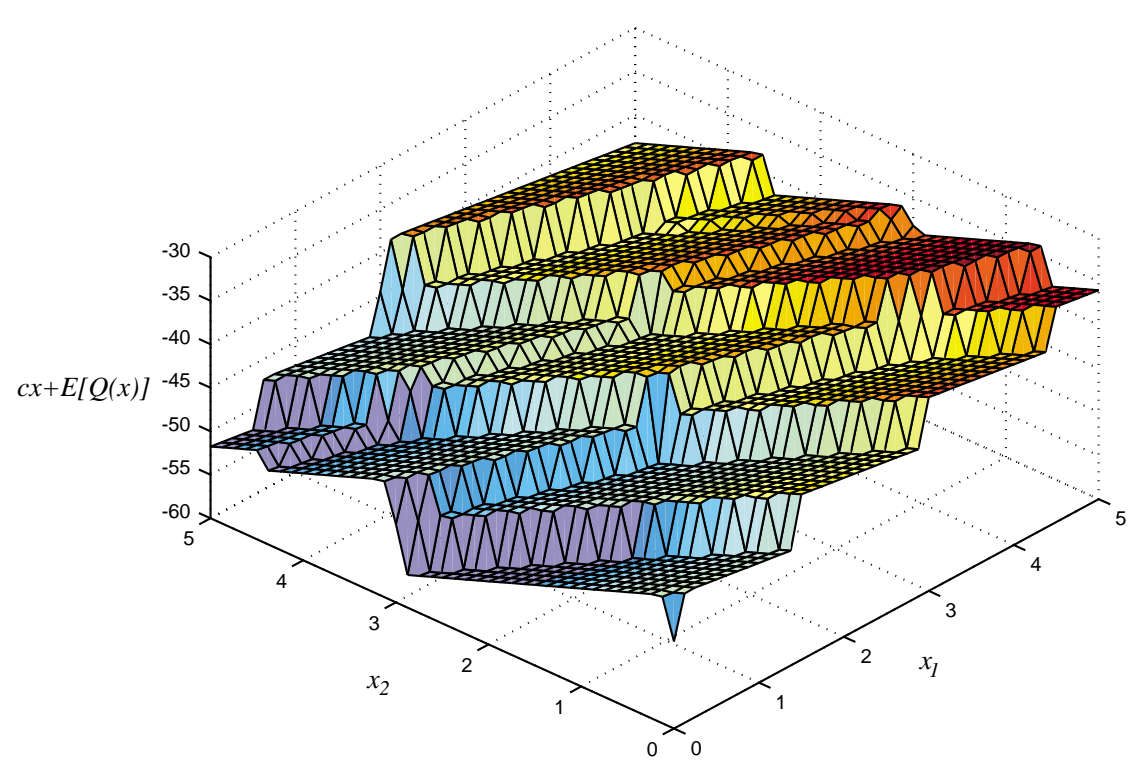

Fig. 1. Value function of EX1.

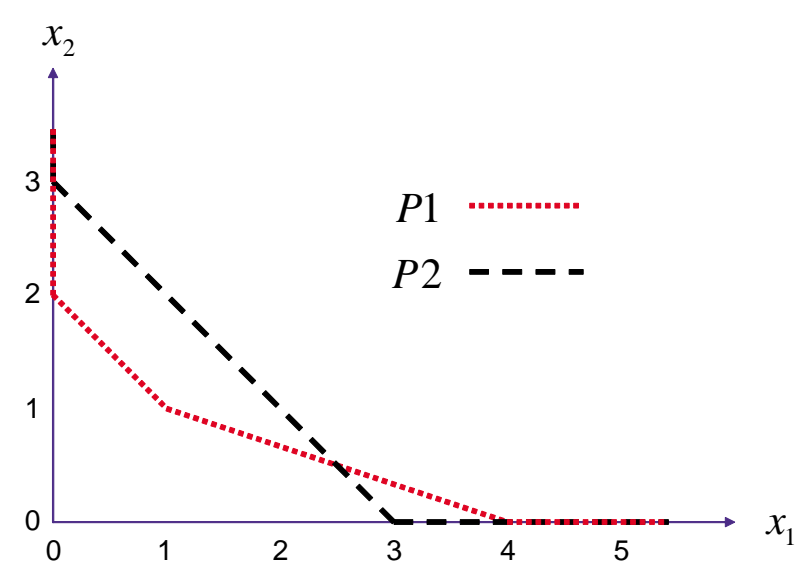

Fig. 2. Feasible set of EX2.

$P 1$. Similarly, let $P 2$ denote the polyhedral set of points satisfying $x_{1}+x_{2} \geq 3$ and $x_{1}+3 x_{2} \geq 0$. The union of $P 1$ and $P 2$ provides the complete feasible set of EX2. This union is not convex as shown in Fig. 2. A similar example is provided by Sen and Higle (1999).

\section{Conclusions}

Several modeling frameworks have been proposed in the literature for optimization under uncertainty. Along with them, a variety of algorithms have been developed and used successfully in many applications.

The current state-of-the-art in this field allows approximate solution of very large-scale problems with sampling-based methods and other approximations of the cost functions. Exact solution of deterministic equivalents is much harder and requires the use of advanced computer architectures.
There are several challenges and opportunities in the area of optimization under uncertainty. Here, we mention a few:

- There is a notable need for systematic comparisons between the different modeling philosophies. A small step in this direction has been taken by Liu and Sahinidis (1996) who compared stochastic programming and fuzzy programming as applied to chemical process planning.

- While significant progress has been made towards the solution of two-stage stochastic programs, the multi-stage case represents a significant challenge. In the case of stochastic integer programming with integer variables in stages other than the first, this represents a conceptual in addition to computational challenge. Deeper understanding of problem structure and properties is required in order to devise applicable algorithms.

- Contrary to the linear case, the integer and non-linear cases have received limited attention. Computational results abound for the linear case but are somewhat limited for the integer and non-linear cases. It appears unlikely that general-purpose algorithms will solve such problems exactly. Instead, we anticipate the development of problem-specific approximation schemes for integer and non-linear problems such as the asymptotically optimal approximation scheme recently proposed by Ahmed and Sahinidis (2003) for capacity expansion of chemical processes.

- In the past, stochastic dynamic programming was used mostly to obtain closed-form solutions of analytically tractable models and numerical solutions to relatively small problem instances. With the recent developments in approximations, especially neuro-dynamic programming, this methodology offers the potential of dealing with problems that for a long time were considered intractable due to either a large state space or the lack of an accurate model. 
- As Section 6 illustrated, there are several opportunities for the development and application of global optimization algorithms to solve optimization problems under uncertainty.

\section{Acknowledgements}

I am grateful to my former students Ming Long Liu and Shabbir Ahmed who introduced me to many of the topics discussed in this paper. Wei Xie read the manuscript and provided useful comments. Thanks are due to an anonymous referee for comments that helped increase the subjects covered in this paper. Partial financial support from the ExxonMobil Upstream Research Company and the National Science Foundation under award DMI 01-15166 is also gratefully acknowledged.

\section{References}

Acevedo, J., \& Pistikopoulos, E. N. (1998). Stochastic optimization based algorithms for process synthesis under uncertainty. Computers \& Chemical Engineering, 22, 647-671.

Adams, W. P., \& Sherali, H. D. (1990). Linearization strategies for a class of zero-one mixed integer programming problems. Operations Research, 38, 217-226.

Ahmed, S. (2002). Strategic planning under uncertainty: Stochastic integer programming approaches. Ph.D. thesis, University of Illinois, Urbana, IL.

Ahmed, S., King, A., \& Parija, G. A. (2003). A multi-stage stochastic integer programming approach for capacity expansion under uncertainty. Journal of Global Optimization, 26, 3-24.

Ahmed, S., \& Sahinidis, N. V. (1998). Robust process planning under uncertainty. Industrial \& Engineering Chemistry Research, 37, 18831892.

Ahmed, S., \& Sahinidis, N. V. (2000). Analytical investigations of the process planning problem. Computers \& Chemical Engineering, 23, $1605-1621$.

Ahmed, S., \& Sahinidis, N. V. (2003). An approximation scheme for stochastic integer programs arising in capacity expansion. Operations Research, 51, 461-471.

Ahmed, S., Tawarmalani, M., \& Sahinidis, N. V. (2003). A finite branch-and-bound algorithm for two-stage stochastic integer programs. Mathematical Programming.

Anderson, E. G. (2001). The nonstationary staff-planning problem with business cycle and learning effects. Management Science, 47, 817832.

Bai, D., Carpenter, T. J., \& Mulvey, J. M. (1997). Making a case for robust models. Management Science, 43, 895-907.

Balas, E., Ceria, S., \& Cornuèjols, G. (1993). A lift and project cutting plane algorithm for mixed 0-1 programs. Mathematical Programming, 58, 295-324.

Bansal, V., Perkins, J. D., \& Pistikopoulos, E. N. (1998). Flexibility analysis and design of dynamic processes with stochastic parameters. Computers \& Chemical Engineering, 22S, S817-S820.

Bastin, F. (2001). Nonlinear stochastic programming. Ph.D. thesis, Department de Mathematique, Faculte des Sciences, Facultes Universitaires Notre-Dame de la Paix, Namur, Belgium.

Beale, E. M. L. (1955). On minimizing a convex function subject to linear inequalities. Journal of the Royal Statistical Society, 17B, 173-184.

Bellman, R. E. (1957). Dynamic programming. Princeton, PA: Princeton University Press.
Bellman, R., \& Zadeh, L. A. (1970). Decision-making in a fuzzy environment. Management Science, 17, 141-161.

Ben-Gal, I., \& Caramanis, M. (2002). Sequential DOE via dynamic programming. IIE Transactions, 34, 1087-1100.

Beraldi, P., Musmanno, R., \& Triki, C. (1998). Solving optimal power dispatch via stochastic linear programming with restricted recourse. Technical report, Department of Electronics, Informatics and Systems, University of Calabria, Rende, Italy.

Berman, O., Ganz, Z., \& Wagner, J. M. (1994). A stochastic optimization model for planning capacity expansion in a service industry under uncertain demand. Naval Research Logistics, 41, 545-564.

Bertsekas, D. P., \& Castañon, D.A. (1999). Rollout algorithms for stochastic scheduling problems. Journal of Heuristics, 5, 89-108.

Bertsekas, D. P., \& Tsitsiklis, J. N. (1996). Neuro-dynamic programming. Belmont, MA: Athena Scientific.

Bienstock, D., \& Shapiro, J. F. (1988). Optimizing resource acquisition decisions by stochastic programming. Management Science, 34, 215229.

Birge, J. R. (1985). Decomposition and partitioning methods for multistage stochastic linear programs. Operations Research, 33, 989-1007.

Birge, J. R. (1997). Stochastic programming computation and applications. INFORMS Journal on Computing, 9, 111-133.

Birge, J. R., \& Dempster, M. A. H. (1996). Stochastic programming approaches to stochastic scheduling. Journal of Global Optimization, 9(3-4), 417-451.

Birge, J. R., \& Louveaux, F. V. (1997). Introduction to stochastic programming. New York, NY: Springer.

Birge, J. R., \& Louveaux, F. V. (1988). A multicut algorithm for two-stage stochastic linear programs. European Journal of Operational Research, 34(3), 384-392.

Birge, J. R., \& Rosa, C. H. (1996). Incorporating investment uncertainty into greenhouse policy models. The Energy Journal, 17, 79-90.

Bit, A. K., Bisswal, M. P., \& Alam, S. S. (1993a). An additive fuzzy programming model for multiobjective transportation problem. Fuzzy Sets and Systems, 57, 183-194.

Bit, A. K., Bisswal, M. P., \& Alam, S. S. (1993b). Fuzzy programming approach to multiobjective solid transportation problem. Fuzzy Sets and Systems, 57, 313-319.

Bitran, G. R., Caldentey, R., \& Mondschein, S. (1998). Coordinating clearance markdown sales of seasonal products in retail chains. $O p$ erations Research, 46, 609-624.

Bitran, G. R., Haas, E. A., \& Matsuo, H. (1986). Production planning of style goods with high setup costs and forecast revisions. Operations Research, 34, 226-236.

Carino, D. R., \& Ziemba, W. T. (1998). Formulation of the Russell-Yasuda Kasai financial planning model. Operations Research, 46, 433-449.

Carøe, C. C. (1998). Decomposition in stochastic integer programming. Ph.D. thesis, University of Copenhagen, Copenhagen, Denmark.

Carøe, C. C., Ruszczyński, A., \& Schultz, R. (1997). Unit commitment under uncertainty via two-stage stochastic programming. In D. Pisinger, C. C. Carøe, \& J. M. Rygaard (Eds.), Proceedings of NOAS 97 (pp. 21-30), Department of Computer Science, University of Copenhagen, Copenhagen, Denmark.

Carøe, C. C., \& Schultz, R. (1999). Dual decomposition in stochastic integer programming. Operations Research Letters, 24, 37-45.

Carøe, C. C., \& Tind, J. (1997). A cutting-plane approach to mixed 0-1 stochastic integer programs. European Journal of Operational Research, 101, 306-316.

Carøe, C. C., \& Tind, J. (1998). L-shaped decomposition of two-stage stochastic programs with integer recourse. Mathematical Programming, 83, 451-464.

Chalam, G. A. (1994). Fuzzy goal programming (FGP) approach to a stochastic transportation problem under budgetary constraint. Fuzzy Sets and Systems, 66, 293-299.

Chanas, S., Kolodziejczyk, W., \& Machaj, A. (1984). A fuzzy approach to the transportation problem. Fuzzy Sets and Systems, 13, 211-221. 
Chanas, S., \& Kuchta, D. (1998). Fuzzy integer transportation problem. Fuzzy Sets and Systems, 98, 291-298.

Chang, N. B., Chen, H. W., Shaw, D. G., \& Yang, C. H. (1997). Water pollution control in river basin by interactive fuzzy interval multiobjective programming. Water Science and Technology, 123, 1208-1216.

Charnes, A., \& Cooper, W. W. (1959). Chance-constrained programming. Management Science, 6, 73-79.

Charnes, A., Cooper, W. W., \& Symonds, G. H. (1958). Cost horizons and certainty equivalents: An approach to stochastic programming of heating oil. Management Science, 6, 235-263.

Chen, V. C. P., Gunther, D., \& Johnson, E. L. (2003). Solving for an optimal airline yield management policy via statistical learning. Journal of the Royal Statistical Society Series C-Applied Statistics, $52,19-30$.

Cheng, L., Subrahmanian, E., \& Westerberg, A. W. (2003). Design and planning under uncertainty: Issues on problem formulation and solution. Computers \& Chemical Engineering, 27, 781-801.

Clay, R. L., \& Grossmann, I. E. (1997). A disaggregation algorithm for the optimization of stochastic planning models. Computers \& Chemical Engineering, 21, 751-774.

Danielsson, A. (2002). Efficiency of catch and effort quotas in the presence of risk. Journal of Environmental Economics and Management, 43, 20-33.

Dantzig, G. B. (1955). Linear programming under uncertainty. Management Science, 1, 197-206.

Davis, M. H. A., Dempster, M. A. H., Sethi, S. P., \& Vermes, D. (1987). Optimal capacity expansion under uncertainty. Advances in Applied Probability, 19, 156-176.

Delgado, M., Herrera, F., Verdegay, J. L., \& Vila, M. A. (1993). Postoptimality analysis on the membership function of a fuzzy linear programming problem. Fuzzy Sets and Systems, 53, 289-297.

Dempster, M. A. H. (1982). A stochastic approach to hierarchical planning and scheduling. In M. A. H. Dempster, et al. (Eds.), Deterministic and stochastic scheduling (pp. 271-296). Dordrecht, The Netherlands: Riedel.

Dempster, M. A. H., Fisher, M. L., Jansen, L., Lageweg, B. J., Lenstra, J. K., \& Rinnooy Kan, A. H. G. (1981). Analytical evaluation of hierarchical planning systems. Operations Research, 29, 707-716.

Dempster, M. A. H., Fisher, M. L., Jansen, L., Lageweg, B. J., Lenstra, J. K., \& Rinnooy Kan, A. H. G. (1983). Analysis of heuristics for stochastic programming: Results for hierarchical scheduling problems. Mathematics of Operations Research, 8, 525-537.

Dert, C. L. (1995). Asset liability management for pension funds: A multistage chance constrained programming approach. $\mathrm{Ph} . \mathrm{D}$. thesis, Erasmus University, Rotterdam, The Netherlands.

Dua, V., Bozinis, N. A., \& Pistikopoulos, E. N. (2002). A multiparametric programming approach for mixed-integer quadratic engineering problems. Computers \& Chemical Engineering, 26, 715-733.

Dupačová, J., Gaivoronski, A., Kos, Z., \& Szantai, T. (1991). Stochastic programming in water management: A case study and a comparison of solution techniques. European Journal of Operational Research, $52,28-44$.

Eppen, G. D., Martin, R. K., \& Schrage, L. (1989). A scenario approach to capacity planning. Operations Research, 37, 517-527.

Ermoliev, Y. (1983). Stochastic quasigradient methods and their application to systems optimization. Stochastics, 9, 1-36.

Escudero, J., Kamesam, P. V., King, A., \& Wets, R. J.-B. (1993). Production planning via scenario modeling. Annals of Operations Research, 43, 311-335.

Ferguson, A., \& Dantzig, G. B. (1956). The allocation of aircraft to routes: An example of linear programming under uncertain demands. Management Science, 3, 45-73.

Floudas, C. A., Gümüç, Z.H., \& Ierapetritou, M. G. (2001). Global optimization in design under uncertainty: Feasibility test and flexibility index problems. Industrial \& Engineering Chemistry Research, 40, 4267-4282.
Furman, K., \& Sahinidis, N. V. (2003). Approximation algorithms for the minimum number of matches problem in heat exchanger network synthesis. Technical report, University of Illinois at Urbana-Champaign.

Gupta, A., \& Maranas, C. D. (2000). A two-stage modeling and solution framework for multisite midterm planning under demand uncertainty. Industrial \& Engineering Chemistry Research, 39, 3799-3813.

Helgason, T., \& Wallace, S. W. (1991). Approximate scenario solutions in the progressive hedging algorithm. A numerical study with an application to fisheries management. Annals of Operations Research, $31,425-444$.

Higle, J. L., \& Sen, S. (1991). Stochastic decomposition: An algorithm for two stage stochastic linear programs with recourse. Mathematics of Operations Research, 16, 650-669.

Higle, J. L., \& Sen, S. (2000). The $C^{3}$ theorem and a $D^{2}$ algorithm for large scale stochastic integer programming: Set convexification. Technical report, University of Arizona, Tuscon.

Horner, P. (1999). Planning under uncertainty. Questions \& answers with George Dantzig. OR/MS Today, 26, 26-30.

Huang, Y. F., Baetz, B. W., Huang, G. H., \& Liu, L. (2002). Violation analysis for solid waste management systems: An interval fuzzy programming approach. Journal of Environmental Management, 65, $431-446$.

IBM Stochastic Solutions. (2003). http://www-3.ibm.com/software/data/ bi/osl/features/stoch-sol.html. Current as of 4 August 2003.

Infanger, G. (1994). Planning under uncertainty: Solving large scale stochastic linear programs. Danvers, MA: Boyd and Fraser Publishing Co.

Infanger, G. (2003). GAMS/Decis user's guide. http://www.gams.com/dd/ docs/solvers/decis.pdf. Current as of 4 August 2003.

Inuiguchi, M., Sakawa, M., \& Kum, Y. (1994). The usefulness of possibilistic programming in production planning problems. International Journal of Production Economics, 33, 45-52.

Kall, P., \& Wallace, S. W. (1994). Stochastic programming. New York, NY: Wiley.

Kallberg, J. G., White, R. W., \& Ziemba, W. T. (1982). Short term financial planning under uncertainty. Management Science, 28, 670-682.

King, A. J., Rockafellar, R. T., Somlyody, L., \& Wets, R. J. -B. (1988). Lake eutrophication management: The lake Balaton project. In $\mathrm{Nu}$ merical techniques for stochastic optimization (vol. 10) (pp. 435-444). Springer Series on Computers and Mathematics. New York, NY: Springer.

King, A. J., Takriti, S., \& Ahmed, S. (1997). Issues in risk modeling for multi-stage systems. Technical report RC-20993, IBM Research Division.

Kirby, D. S., Fiksen, O., \& Hart, P. J. B. (2000). A dynamic optimisation model for the behaviour of tunas at ocean fronts. Fisheries Oceanography, 9, 328-342.

Klein Haneveld, W. K., Stougie, L., \& van der Vlerk, M. H. (1995). On the convex hull of the simple integer recourse objective function. Annals of Operational Research, 56, 209-224.

Klein Haneveld, W. K., Stougie, L., \& van der Vlerk, M. H. (1996). An algorithm for the construction of convex hulls in simple integer recourse programming. Annals of Operational Research, 64, 67-81.

Kouwenberg, R., \& Zenios, S. A. (2001). Stochastic programming models for asset liability management. Technical report, HERMES Center of Excellence on Computational Finance \& Economics, University of Cyprus, Nicosia, Cyprus.

Laguna, M. (1998). Applying robust optimization to capacity expansion of one location in telecommunications with demand uncertainty. Management Science, 44, S101-S110.

Lai, Y.-J., \& Hwang, C.-L. (1993a). A stochastic possibilistic programming model for bank hedging decision problems. Fuzzy Sets and Systems, 57, 351-363.

Lai, Y.-J., \& Hwang, C.-L. (1993b). Possibilistic linear programming for managing interest rate risk. Fuzzy Sets and Systems, 54, 135146. 
Laporte, G., \& Louveaux, F. V. (1993). The integer L-shaped method for stochastic integer programs with complete recourse. Operations Research Letters, 13, 133-142.

Laporte, G., Louveaux, F. V., \& Mercure, H. (1989). Models and exact solutions for a class of stochastic location-routing problems. European Journal of Operational Research, 39, 71-78.

Laporte, G., Louveaux, F. V., \& Mercure, H. (1992). The vehicle routing problem with stochastic travel times. Transportation Science, 26, 161170.

Laporte, G., Louveaux, F. V., \& Mercure, H. (1994). A priori optimization of the probabilistic traveling salesman problem. Operations Research, $42,543-549$.

Laporte, G., Louveaux, F. V., \& van Hamme, L. (1994). Exact solution of a stochastic location problem by an integer L-shaped algorithm. Transportation Science, 28(2), 95-103.

Lenstra, J. K., Rinnooy Kan, A. H. G., \& Stougie, L. (1983). A framework for the probabilistic analysis of hierarchical planning systems. Technical report, Mathematisch Centrum, University of Amsterdam, Amsterdam, The Netherlands.

Li, L. S., \& Lai, K. K. (2000). A fuzzy approach to the multiobjective transportation problem. Computers \& Operations Research, 27, 43-57.

Linderoth, J., Shapiro, A., \& Wright, S. (2002). The empirical behavior of sampling methods for stochastic programming. Technical report, University of Wisconsin, Madison, WI.

Liu, M. L., \& Sahinidis, N. V. (1996). Optimization in process planning under uncertainty. Industrial \& Engineering Chemistry Research, 35, 4154-4165.

Liu, M. L., \& Sahinidis, N. V. (1997a). Bridging the gap between heuristics and optimization: The capacity expansion case. AIChE Journal, 43, 2289-2299.

Liu, M. L., \& Sahinidis, N. V. (1997b). Process planning in a fuzzy environment. European Journal of Operational Research, 100, 142169

Louveaux, F. V. (1980). A solution method for multistage stochastic programs with recourse with application to an energy investment problem. Operations Research, 28(4), 889-902.

Malcolm, S. A., \& Zenios, S. A. (1994). Robust optimization of power systems capacity expansion under uncertainty. Journal of the Operational Research Society, 45, 1040-1049.

Miller, W. A., Leung, L. C., Azhar, T. M., \& Sargent, S. (1997). Fuzzy production planning model for fresh tomato packing. International Journal of Production Economics, 53, 227-238.

Mo, B., Gjelsvik, A., \& Grundt, A. (2001). Integrated risk management of hydro power scheduling and contract management. IEEE Transactions on Power Systems, 16, 216-221.

Morton, D. P. (1996). An enhanced decomposition algorithm for multistage stochastic hydroelectric scheduling. Annals of Operations Research, 64, 211-235.

Mulvey, J. M., Vanderbei, R. J., \& Zenios, S. A. (1995). Robust optimization of large-scale systems. Operations Research, 43, 264-281.

Mulvey, J. M., \& Vladimirou, H. (1992). Stochastic network programming for financial planning problems. Management Science, 38, 1642-1664.

Nakayama, H., \& Kagaku, N. (1998). Pattern classification by linear goal programming and its extensions. Journal of Global Optimization, 12, $111-126$.

Nayak, R. C., \& Panda, R. K. (2001). Integrated management of a canal command in a river delta using multi-objective techniques. Water Resources Management, 15, 383-401.

Norkin, V. I., Ermoliev, Y. M., \& Ruszczyński, A. (1998). On optimal allocation of indivisibles under uncertainty. Operations Research, 46, 381-395.

Parra, M. A., Terol, A. B., \& Uria, M. V. R. (2001). A fuzzy goal programming approach to portfolio selection. European Journal of Operational Research, 133, 287-297.

Pereira, M. V. F., \& Pinto, L. M. V. G. (1991). Multi-stage stochastic optimization applied to energy planning. Mathematical Programming, $52,359-375$.
Pickenss, J. B., \& Hof, J. G. (1991). Fuzzy goal programming in forestry: An application with special solution problems. Fuzzy Sets and Systems, 39, 239-246.

Pinter, J. (1991). Stochastic modelling and optimization for environmental management. Annals of Operational Research, 31, 527-544.

Pistikopoulos, E. N., \& Grossmann, I. E. (1989a). Optimal retrofit design for improving process flexibility in nonlinear systems. I. Fixed degree of flexibility. Computers \& Chemical Engineering, 13, 1003-1016.

Pistikopoulos, E. N., \& Grossmann, I. E. (1989b). Optimal retrofit design for improving process flexibility in nonlinear systems. II. Optimal level of flexibility. Computers \& Chemical Engineering, 13, 1087-1096.

Pistikopoulos, E. N., \& Mazzuchi, T. A. (1990). A novel flexibility analysis approach for processes with stochastic parameters. Computers \& Chemical Engineering, 14, 991-1000.

Prékopa, A. (1971). Logarithmic concave measures with applications to stochastic programming. Acta Scientifica Mathematica (Szeged), 32, 301-316.

Prékopa, A. (1995). Stochastic programming. Dordrecht, The Netherlands: Kluwer Academic Publishers.

Richter, S., Vandezande, K., Chen, N., Zhang, K., Sutherland, J., Anderson, J., Han, L. P., Panton, R., Branco, P., \& Gallie, B. (2003). Sensitive and efficient detection of $R B 1$ gene mutations enhances care for families with retinoblastoma. American Journal of Human Genetics, 72, 253 269.

Rockafellar, R. T., \& Wets, R. J.-B. (1991). Scenarios and policy aggregation in optimization under uncertainty. Mathematics of Operations Research, 16(1), 119-147.

Ruszczyński, A. (1986). A regularized decomposition method for minimizing a sum of polyhedral functions. Mathematical Programming, 35, 309-333.

Sahinidis, N. V. (2003). Optimization under uncertainty: State-of-the-art and opportunities. In I. E. Grossmann, \& C. McDonald (Eds.), Proceedings of Foundations of Computer-Aided Process Operations (FO$C A P O$ 2003): A view to the future integration of $R \& D$, manufacturing and the global supply chain, CACHE Corporation, Austin, TX.

Sakawa, M., Nishizaki, I., \& Uemura, Y. (2002). A decentralized two-level transportation problem in a housing material manufacturer: Interactive fuzzy programming. European Journal of Operational Research, 141, 167-185.

Schultz, R., Stougie, L., \& van der Vlerk, M. H. (1998). Solving stochastic programs with integer recourse by enumeration: A framework using Gröbner basis reductions. Mathematical Programming, 83, 229-252.

Sen, S., \& Higle, J. (1999). An introductory tutorial on stochastic linear programming. Interfaces, 29, 33-61.

Sernland, E., Olsson, A., \& Holmgren, N. M. A. (2003). Does information sharing promote group foraging? Proceedings of the Royal Society of London Series B-Biological Sciences, 270, 1137-1141.

Shapiro, A., \& Homem-de-Mello, T. (1998). A simulation-based approach to two-stage stochastic programming with recourse. Mathematical Programming, 81, 301-325.

Shapiro, A., \& Wardi, Y. (1996). Convergence analysis of gradient descent stochastic algorithms. Journal of Optimization Theory and Applications, 91, 439-454.

Shen, Y. X., \& Leachman, R. C. (2003). Stochastic wafer fabrication scheduling. IEEE Transactions on Semiconductor Manufacturing, 16, $2-14$.

Sherali, H. D., \& Fraticelli, B. M. P. (2002). A modified Benders' partitioning approach for discrete subproblems: An approach for stochastic programs with integer recourse. Journal of Global Optimization, 22, 319-342.

Sherali, H. D., Soyster, A. L., Murphy, F. H., \& Sen, S. (1984). Allocation of capital costs in electric utility capacity expansion planning under uncertainty. Management Science, 30, 1-19.

Shukla, A. K., \& Gupta, S. N. (1989). A stochastic linear programming approach for crop planning. Acta Ciencia Indica Mathematics, 15, 265-270. 
Slowinski, R. (1986). A multicriteria fuzzy linear programming method for water supply system development planning. Fuzzy Sets and Systems, 19, 217-237.

Somlyody, L., \& Wets, R. J.-B. (1988). Stochastic optimization models for lake eutrophication management. Operations Research, 36, 660-681.

Spaccamela, A. M., Rinnooy Kan, A. H. G., \& Stougie, L. (1984). Hierarchical vehicle routing problems. Networks, 14, 571-586.

Stochastic Programming Community Home Page. (2003). http://stoprog. org. Current as of 4 August 2003.

Stougie, L. (1985). Design and analysis of methods for stochastic integer programming. Ph.D. thesis, University of Amsterdam, Amsterdam, The Netherlands.

Straub, D. A., \& Grossmann, I. E. (1993). Design optimization of stochastic flexibility. Computers \& Chemical Engineering, 17, 339-354.

Swaney, R. E., \& Grossmann, I. E. (1985). An index for operational flexibility in chemical process design. Part 1. Formulation and theory. AIChE Journal, 31, 621-630.

Takriti, S., \& Ahmed, S. (2003). On robust optimization of two-stage systems. Mathematical Programming.

Takriti, S., Birge, J. R., \& Long, E. (1996). A stochastic model of the unit commitment problem. IEEE Transactions on Power Systems, 11, $1497-1508$.

Tanaka, H., \& Asai, K. (1984). Fuzzy linear programming problems with fuzzy numbers. Fuzzy Sets and Systems, 13, 1-10.

Tanaka, H., Okuda, T., \& Asai, K. (1974). On fuzzy mathematical programming. Journal of Cybernetics, 3, 37-46.

Tawarmalani, M., \& Sahinidis, N. V. (2002). Convexification and global optimization in continuous and mixed-integer nonlinear programming: Theory, algorithms, software, and applications. Nonconvex optimization and its applications (vol. 65). Dordrecht, The Netherlands: Kluwer Academic Publishers.

Tayur, S. R., Thomas, R. R., \& Natraj, N. R. (1995). An algebraic geometry algorithm for scheduling in the presence of setups and correlated demands. Mathematical Programming, 69, 369-401.

Tintner, G. (1955). Stochastic linear programming with applications to agricultural economics. In H. A. Antosiewicz (Ed.), Proceedings of the Second Symposium in Linear Programming (pp. 197-228), National Bureau of Standards, Washington, DC.

Tomasgard, A., Dye, S., Wallace, S., Audestad, J., Stougie, L., \& van der Vlerk, M. (1998). Modelling aspects of distributed processing in telecommunication networks. Annals of Operations Research, 82, 161-184.

Tong, S. (1994). Interval number and fuzzy number linear programming. Fuzzy Sets and Systems, 66, 301-306.

Tsitsiklis, J. N., \& Van Roy, B. (1999). Optimal stopping of Markov processes: Hilbert space theory, approximation algorithms, and an application to pricing financial derivatives. IEEE Transactions on Automatic Control, 44, 1840-1851.

Van Roy, B., \& Tsitsiklis, J. N. (2001). Regression methods for pricing complex American-style options. IEEE Transactions on Neural Networks, 12, 694-703.

Van Slyke, R., \& Wets, R. J.-B. (1969). L-shaped linear programs with applications to optimal control and stochastic programming. SIAM Journal on Applied Mathematics, 17, 638-663.

Verweij, B., Ahmed, S., Kleywegt, A., Nemhauser, G., \& Shapiro, A. (2002). The sample average approximation method applied to stochastic routing problems: A computational study. Technical report, Georgia Institute of Technology, Atlanta, GA.

Wagner, J. M., Shamir, U., \& Marks, D. H. (1994). Containing groundwater contamination: Planning models using stochastic programming with recourse. European Journal of Operational Research, 77, 1-26.

Watanabe, T., \& Ellis, H. (1993). Stochastic programming models for air quality management. Computers \& Operations Research, 20, 651-663.

Wets, R. J.-B. (1966). Programming under uncertainty: The solution set. SIAM Journal on Applied Mathematics, 14, 1143-1151.

Wets, R. J.-B. (1974). Stochastic programs with fixed recourse: The equivalent deterministic program. SIAM Review, 16, 309-339.

Wu, S. M., Huang, G. H., \& Guo, H. C. (1997). An interactive inexactfuzzy approach for multiobjective planning of water resource systems. Water Science and Technology, 36, 235-242.

Yalcin, C., \& Stott, A. W. (2000). Dynamic programming to investigate financial impacts of mastitis control decisions in milk production systems. Journal of Dairy Research, 67, 515-528.

Zimmerman, F. J., \& Carter, M. R. (2003). Asset smoothing, consumption smoothing and the reproduction of inequality under risk and subsistence constraints. Journal of Development Economics, 71, 233-260.

Zimmermann, H.-J. (1978). Fuzzy programming and linear programming with several objective functions. Fuzzy Sets and Systems, 1, 45-55.

Zimmermann, H.-J. (1991). Fuzzy set theory and its application (2nd ed.). Boston: Kluwer Academic Publishers. 